\title{
Retail crowding: meta-analysis of contextual and cultural moderators
}

Fernando_de Oliveira Santini_(Universidade do Vale do Rio dos Sinos, São Leopoldo, Brazil)

Wagner Junior_Ladeira_(Universidade do Vale do Rio dos Sinos, São Leopoldo, Brazil) Diego Costa_Pinto_(NOVA Information Management School (NOVA IMS), Universidade Nova de Lisboa, Campus de Campolide, Lisboa, Portugal)

Marcia Maurer_Herter_(Universidade Europeia, Business and Law Research Center, UNIDCOM, Lisboa, Portugal)

Anna S._Mattila__Hospitality Management, Pennsy/vania State University, University Park, Pennsy/vania, USA)

Marcelo Gattermann_Perin_(FGV, São Paulo, Brazil)

This is the accepted author manuscript of the following article published by EMERALD:

De Oliveira Santini, F., Ladeira, W. J., Pinto, D. C., Herter, M. M., Mattila, A. S., \& Perin, M. G. (2021). Retail crowding: meta-analysis of contextual and cultural moderators. Marketing Intelligence and Planning. [Advanced online publication on 1 November 2021]. https://doi.org/10.1108/MIP-03-2021-0076

\section{Funding:}

The study has been funded by FUNDAÇÃO DE AMPARO À PESQUISA DO ESTADO DO RIO GRANDE DO SUL (FAPERGS) (Award Number: 19/2551-0001794-4)

This work is licensed under a Creative Commons Attribution-NonCommercial 4.0 International License. 


\title{
Retail Crowding: Meta-Analysis of Contextual and Cultural Moderators
}

\author{
Abstract \\ Purpose: Although academics and retail managers share a common belief that crowded stores \\ generate more sales, there is a growing concern about the negative impact of retail crowding \\ on customer relationship management. This research aims to understand the underlying \\ processes driving the effect and it explores potential moderators that may mitigate the \\ negative effects on consumer satisfaction. \\ Design/methodology/approach: This research employs a meta-analysis on retail crowding \\ effects and potential moderators. \\ Findings: Our integrative model of retail crowding reveals that social needs, crowd \\ similarity, crowd expectation, and uncertainty avoidance mitigate the negative retail crowding \\ effects on satisfaction. \\ Research limitations/implications: We advance the retailing literature by synthesizing \\ recent studies on retail crowding. Our findings also provide a clearer understanding of the \\ mediating role of negative emotions in the relationship between retail crowding and \\ satisfaction.
}

Practical implications: This research offers guidance for retail managers on how to mitigate the harmful effects of crowding on customer satisfaction.

Originality: This research contributes to the retailing literature and offers guidance for retailers on how to mitigate the harmful effects of crowding on customer satisfaction. Our moderation analyses provide insights into how and when crowding drives consumer satisfaction.

Keywords: retail crowding, satisfaction, negative emotions, meta-analysis, moderators. 


\section{Introduction}

There is a common belief among retailers that crowded stores generate more sales. This assumption is well supported by decades of academic research demonstrating the link between crowding and sales performance (Harrell et al., 1980, 2013; Hock and Bagchi, 2017). However, there is a growing concern about the negative effects of crowding in retail environments (Blut and Iyer, 2020; Das and Varshneya, 2017; Eroglu et al., 2005). Evidence from both laboratory and field studies indicates that retail crowding is a growing challenge for customer relationship management (CRM) (Demoulin and Willems, 2019; Mehta, 2013).

Crowding can generate negative emotions and reduced satisfaction (Dahm et al., 2018; Demoulin and Willems, 2019), promoted by long queues, messy shelves, lack of sales personnel, parking hassles, loud ambient music, or service failures (Mehta, 2013). Recently, consumers' concerns with health and safety issues have dramatically reduced the physical presence of customers in stores and accentuated the negative crowding effects (Wharton, 2020)

The main goal of this study is to understand the underlying process of negative emotions explaining the retail crowding effect and to explore the impact of possible moderators that may mitigate the negative effect of crowding on consumer satisfaction. Our integrative theoretical model of retail crowding contributes to the literature in several ways. First, we provide a more nuanced understanding of the mediating role of negative emotions in the relationship between retail crowding and satisfaction. Second, our moderation analyses offer insight into how and when crowding drives consumer satisfaction. Taken together, our meta-analytical approach answers the call for research on crowding regarding consumer uncertainty (Krohne et al., 1992; Machleit et al., 2000), indulgence (Bressolles et al., 2007; Hock and Bagchi, 2017), social factors (Baker and Wakefield, 2012; Demoulin and Willems, 
2019), perceived similarity of shoppers (Lucia-Palacios et al., 2016, 2018), crowding expectations (Machleit et al., 2000; Thomas and Saenger, 2019), and time pressure during decision-making (Machleit et al., 2000; Pan and Siemens, 2011).

Moreover, we synthesize findings from recent studies on retail crowding to offer a more comprehensive understanding of its effects on consumer satisfaction. Although we acknowledge the importance of previous studies examining retail crowding, there is a need for further investigation. Metha (2013) offered a critical review of the perceived retail crowding and future research on the topic. Wumei et al (2020) and Santini (2020 empirically examined the crowding effects but they failed to offer a theoretical model integrating the effects. Finally, Blut and Iyer (2020) proposed a theoretical model but they did not test important moderators such as crowd similarity (Pons et al., 2016; Dahm et al., 2018), culture (Metha, 2013; Das and Varshneya, 2017; Blut and Iyer, 2020), and social needs (Metha, 2013). This research extends previous studies on retail crowding by using a robust meta-analytical procedure (Meta-Analytic Structural Equation Modeling - MASEM) to examine relevant moderators, thus contributing to the retailing literature. We also offer guidance for retail managers on how to mitigate the harmful effects of crowding on customer satisfaction.

\section{The retail crowding debate:}

The retailing literature emerged in the 70's when super stores and shopping centers became popular (Harrell and Hutt, 1976; Metha, 2013). Early studies used theories from social and environmental psychology to better understand crowding effects from a lens of excessive stimulation (Desor, 1972), a dysfunctional dense sensation (Stokols et al., 1973) or perceived privacy invasion (Altman, 1975). 
The literature further evolved by differentiating between (a) human or social density and (b) spatial density (Mehta 2013). The human/social density reflects the number of people in the shopping environment (Machleit el al., 2000) and focuses on queuing (Dahm et al., 2018), noise (Nagar et al., 1987) and locus of control (Eroglu and Machleit, 1990). The spatial density, on the other hand, reflects the physical space available to shoppers in the retail store (Metha, 2013). Such studies tend to focus on the impact of the store's physical characteristics, such store size and layout, on perceived crowding (Park and Zhang, 2019).

Perceived crowding comprises "the consumer's evaluative assessment of restrictive aspects of the retail space" (Meta, 2013, p. 643). Unlike the density dimension, this definition is more subjective and suggests that a number of factors might influence consumers' crowding perceptions, including personal (Machleit et al., 2000), cultural (Pons and Laroche, 2007) and situational factors (Eroglu and Machleit, 1990). In terms of measurement issues, some researchers used unidimensional constructs (e.g., Eroglu and Machleit, 1990) while others employed a two-dimensional approach (e.g., Machleit et al., 2000).

Recent meta-analyses attempted to gain a deeper understanding of the crowding phenomenon. However, such studies (Wumei et al., 2020; Santini et al., 2020) failed to test a theoretically-driven model. In this paper, we present a theoretical model that captures the crowding effect on customer satisfaction using the Meta-Analytic Structural Equation Modeling (MASEM). In addition, we examine several possible moderators related to (1) contextual and (2) cultural factors.

\section{Insert Figure 1}

Table 1 presents the constructs used in the theoretical model. This meta-analysis used a systematic review to identify theoretically relevant constructs. We then selected the 
constructs with a minimum of three effect sizes as recommended by Hunter and Schmidt (2004).

\section{Insert Table 1}

\subsection{Retail Crowding Framework}

The link between retail crowding, negative emotions and customer satisfaction is an ongoing challenge for both academic researchers and practitioners (Mehta, 2013; Pons et al., 2016; Das and Varshneya, 2017; Demoulin and Willems, 2019). The pressing question is to better understand how to enhance customer satisfaction in crowded retail environments.

Previous research provides plenty of evidence for the consequences of crowding (e.g., Pons et al., 2014; Machleit et al., 2000; Mehta, 2013; Park and Zhang, 2019). However, contextual factors might influence consumers' tolerance for crowding (e.g., Eroglu et al., 2005). For example, Byun and Sternquist (2012) show that specific events such as clearance sales and grand openings are expected to attract a lot of shoppers. Moreover, crowding has been shown to have a positive influence on satisfaction with certain retail environments such as restaurants and shopping centers (e.g., Aguilar, 2014) and contexts that involve high levels of social interaction (e.g., stadiums, bars, and clubs; Yoshida and James, 2010). Although prior research yields mixed findings, the overwhelming evidence suggests a negative effect of retail crowding on customer satisfaction. Therefore, we put forth the following prediction.

H1. Retail crowding will have a negative effect on customer satisfaction. 
Companies invest considerable resources into creating attractive shopping environments (Pons et al., 2014, Thomas and Saenger, 2019). Nevertheless, crowded shopping environments can induce negative emotions due to loud ambient music, messy shelves, service failures, parking hassles, long queues or lack of personnel (Lucia-palacios et al., 2018). Negative emotions induced by crowded environments (Dion, 2004; Machleit et al., 2000) tend to evoke avoidance behaviors (Knoeferle et al., 2017), stress (Baker and Wakefield, 2011), and anxiety (Gelbrich and Sattler, 2014). In other words, retail crowding can generate negative emotions, which in turn, lead to reduced customer satisfaction (Mehta, 2013). Therefore, we propose that negative emotions mediate the relationship between retail crowding and customer satisfaction.

H2. Negative emotions mediate the relationship between retail crowding and customer satisfaction.

\section{Potential Moderators}

\subsection{Contextual Moderators}

First, we analyze four possible contextual moderators: (1) social needs; (2) crowd similarity; (3) expectations; and (4) time pressure. In terms of social factors, individuals modify their behavior in response to social cues, resulting in adaptive social processes (Thomas and Saenger, 2019). Social factors in the store environment, such as the number of people, their appearance and behavior (salespersons and other customers) are important elements that influence customer experiences (Demoulin and Willems, 2019). We assume that social needs reflect people's predisposition to interact with other consumers in retail environments. Accordingly, social needs are linked to consumer responses to crowding (Mehta, 2013). Rampay et al. (2012) demonstrate that consumers with high social needs 
derive more pleasure from crowded contexts and spend more money in dense settings. We thus propose the following:

H3. In crowded retail environments, consumers with low social needs will have higher levels of dissatisfaction than consumers with high social needs.

The second contextual moderator is perceived crowd similarity. Crowd similarity represents the extent to which target customers feel that they are similar to shoppers (LuciaPalacios et al., 2018). Perceived similarity can be identified through behaviors and physical appearance of other customers (Brocato et al., 2012). Some studies suggest that low levels of perceived similarity tend to promote consumer discomfort (Maner et al., 2007), and therefore, magnify the negative effect of retail crowding on customer satisfaction. Moreover, prior retailing studies show a positive relationship between perceived similarity and satisfaction via affective and cognitive processes (e.g., Kwon et al., 2016). People's similarity perceptions tend to induce feelings of comfort, thus leading to a more satisfying shopping experience (Lucia-Palacios et al., 2018). Shopping with similar others makes the activity more entertaining (Lucia-Palacios et al., 2016). We thus put forth the following prediction:

H4. In crowded retail environments, perceived similarity (vs. dissimilarity) with other customers will have a positive impact on customer satisfaction.

The third contextual moderator is crowd expectations for consumption situations or events that are linked to crowding such as Black Friday and Christmas season (Spangenberg et al., 2006). When consumers expect to encounter crowding, the negative relationship between retail crowding and satisfaction is attenuated. Prior research shows that consumers' density expectations and the shopping context interact to make the experience either pleasant or unpleasant (Sinha and Nayyar, 2000). If perceived crowding for a dense event falls short of 
(vs. exceeds) expectations, consumers tend to be less satisfied (Machleit et al. 2000). For example, consumers expect crowding in discount stores, thus having a positive impact on shopping satisfaction (Aguilar, 2014). Thus, we put forth the following prediction:

H5. Consumers with low expectations for crowding will have higher levels of dissatisfaction than their counterparts with high expectations.

The fourth contextual moderator is time pressure. Previous research demonstrates that time pressure affects consumers' crowding perceptions (Mehta, 2013; Pan and Siemens, 2011). Time-constrained consumers tend to be less satisfied in crowded environments as such contexts hinder their shopping goals (Gelbrich and Sattler, 2014). In other words, minimizing the time needed to shop is a priority for time-pressed consumers (Pan and Siemens, 2011). We thus propose that shopping activities involving time pressure will magnify the negative relationship between retail crowding and customer satisfaction.

H6. In crowded retail environments, consumers with time pressure will have higher levels of dissatisfaction than consumers without time pressure.

\subsection{Cultural Moderators}

Understanding how culture shapes reactions to crowded environments is important since cultural values, such as uncertainty avoidance, influence consumer behavior (Mehta, 2013). Uncertainty avoidance refers to the "level of stress in a society in the face of an unknown future" (Hofstede et al., 2011; p. 8). Kim et al., (2010) show that high uncertainty avoidance makes consumers more tolerant of crowded restaurants. In a similar vein, Wong and Lar (2001) demonstrate that in cultures with high uncertainty avoidance (e.g., China), tourists prefer to travel in groups because they feel safer. Therefore, we predict the following: 
H7. In crowded retail environments, consumers in low (vs. high) uncertainty avoidance cultures level will have higher levels of dissatisfaction.

\section{Meta-analytical procedures}

Our procedures follow the PRISMA protocol (Preferred Reporting Items for Systematic Reviews and Meta-Analyzes) (Moher et al., 2009). We performed four steps: (1) the literature search; (2) the inclusion criteria; (3) the coding process; (4) and the MASEM analysis.

Literature search. Search procedures included multiple sources to identify published and unpublished studies (Rosenthal and DiMatteo, 2001). We searched the keywords "crowd", "crowding", "density", "human density" and "spatial density" in the "document title" and/or "summary" fields in the following databases: JSTOR, Emerald, PsycINFO, Taylor \& Francis, Elsevier, Science Direct, SCOPUS, Scielo, and EBSCO. In addition, we manually searched for papers presented in main marketing conferences including the Association for Consumer Research Conference, Academy of Marketing Science Conference, European Marketing Academy Conference, and Global Marketing Conference. Finally, we checked the ProQuest Dissertations \& Theses Global database. The keywords were searched in the titles, abstracts, and lists of keywords of papers published until December 2020. The number of papers initially derived from the search was 912.

Inclusion criteria. We followed the common approach of recent meta-analytic reviews (e.g., Rubera and Kirca, 2012; Babić-Rosario et al. 2016) to identify studies that would be retained for the meta-analysis. To be included, the study must: (1) examine the link between crowding perceptions and/or negative emotions and/or customer satisfaction; (2) measure crowding perceptions in a retail context, and; (3) present statistical results of 
perceived crowding on dependent variable(s) (e.g., correlation; beta; t-test; F-ratio; chisquare). 193 studies were eliminated because they involved a qualitative or a conceptual approach. Additional 536 studies were excluded because they did not examine the relationships of interest in this study (e.g., studies did not measure crowding, human density or spatial density) or they did not involve a retail context. This step also excluded 98 studies that merely presented descriptive statistics. The final sample was composed of 85 studies that reported 679 effects-sizes. For the Meta-Analytic Structural Equation Modeling (MASEM), we only considered studies that presented the relationship between crowding and/or negative emotions and/or customer satisfaction (32 studies with 185 effects-sizes). The number of papers per year on retail crowding is shown in Figure 2.

Insert figure 2

Coding process. The coding process followed Rust and Coil's (1994) suggestions as applied in previous meta-analysis (e.g., Babić-Rosario et al. 2016; Santini et al., 2020). This process was executed by three researchers. Prior to the coding process, all the procedures were discussed by the researchers. In case of a disagreement or doubt, an independent researcher reviewed the study. The inter-rater agreement score was $86 \%$. The main information coded were: (1) the study origin; (2) year of the study; (3) sample size; (4) crowding dimension (e.g., human crowding; spatial crowding; full scale); (5) scale reliability; (6) variable/construct related to crowding perception (e.g., negative emotion and/or customer satisfaction); (7) effect of the relationship between crowding perception and/or negative emotions and/or customer satisfaction. We also coded the moderators as presented in Table 2. 
Analytical procedures. In congruence with previous research (Hedges and Olkin 2014; Hunter and Schmidt(2004), the effect size was captured via Pearson's correlation coefficient (r), and the effect sizes were weighted based on the sample size and scale reliability). If the study did not report correlations but presented alternative statistics (e.g., standardized regression coefficients, F or T-tests) we converted them to a Pearson correlation by following the procedures from Hedges and Olkin (1995). We also took the mean effect size of the two dimensions (i.e., human and spatial crowding) when the study did not report the combined effect. This approach is commonly used in previous meta-analytical studies (e.g., Babić-Rosario et al. 2016; Santini et al., 2020). In the analysis, we applied the random effect method to estimate the effect sizes (Hunter and Schmidt 2004). The random effect model was chosen because it offers more generalizable results to studies with heterogeneous sample sizes (Rosenthal and DiMatteo, 2001).

Our direct and mediation effects were tested by Meta-Analytic Structural Equation Modeling (MASEM). MASEM is a process that integrates meta-analytic covariances into standard structural equations modeling techniques (Cheung 2015; Viswesvaran and Ones, 1995). The analyses were conducted by metaSEM in R (Cheung, 2015) and OpenMx 2.0 (Neale et al., 2016). The correlation matrix is shown in Table 3. Finally, we tested the moderating effects by hierarchical linear meta-analysis (HiLMA), a multivariate regressionbased approach (Geyskens et al. 2009). Finally, the contextual, cultural, and study-specific moderators are presented in Appendix B1 and B2.

Insert Table 3 


\section{Findings}

\subsection{Descriptive results and correlation of crowding and negative emotions and customer}

satisfaction

First, we show two correlational effects identified by MASEM: (1) retail crowding and negative emotions and (2) retail crowding and customer satisfaction. Table 4 presents the main results.

Insert Table 4

The results show a positive and significant effect of retail crowding on negative emotions $(r=.339 ; \mathrm{CI}=.240 / .431)$, with high Fail-Safe Number (Rosenthal, 1991) $(\mathrm{FSN}=$ 167161). The relationship had high heterogeneity $\left(Q=1381.01 ; p<.001 ; \mathrm{I}^{2}=97.3 \%\right)$ (Higgins et al. 2003). Finally, we noted that publication bias did not influence these results, since the Egger regression did not present significant effects (intercept $=4.133, S E=3.505, t$ $=1.178, p=.246)($ Egger et al., 1997).

In terms of the relationship between retail crowding and customer satisfaction we also found a signficant postive effect $(r=-.133 ; \mathrm{CI}=-255 / .006)$, with high Fail-Safe Number $(\mathrm{FSN}=1366)$ and hetorogeneity $\left(Q=651.83 ; p<.001 ; \mathrm{I}^{2}=96.3 \%\right)$. The publication bias, again, did not influence our findings (intercept $=-.747, S E=2.876, t=-.259, p=.797$ ).

\subsection{Direct effects}


The direct relationships were tested by Meta-Analytic Structural Equation Modeling (MASEM). The indices of the final model indicated a good fit (Cheung, 2015) (TLI=.883; $C F I=.981 ; R M S E A=.024)$. The model index is similar to the models presented by Blut and Iyer (2020). Table 5 shows that retail crowding is negatively linked to customer satisfaction $(\beta=.192, L C I=-.307 ; U C I=-.076 ; p<.05)$. In other words, crowding results in negative experiences (Eroglu et al., 2005; Das and Varshneya, 2017), supporting H1. These findings provide empirical evidence for theoretical accounts linking perceived crowding with negative emotions such as anger, stress and contempt (Dion, 2004; Machleit et al., 2000).

Next, we tested the mediating role effect of negative emotions in the relationship between retail crowding and customer satisfaction. The indirect effect is significant $(\beta=-$ $.172, L C I=-.239 ; U C I=-.111 ; p<.001)$. We then examined the direct relationship of retail crowding on customer satisfaction by a saturated model (Chung, 2015; Jak, 2015). The negative direct effect of retail crowding on customer satisfaction decreased $(\beta=-.159, L C I=-$ $.257 ; U C I=-.086 ; p<.05)$, suggesting a partial mediation. These findings provide support for $\mathrm{H} 2$ indicating the impact of retail crowding on negative emotions and reduced customer satisfaction (Mehta, 2013).

\section{Insert Table 5}

\subsection{Moderating effects}

We tested the moderating effects via hierarchical linear meta-analysis (HiLMA). The results are summarized in Figure 3. 
Insert Figure 3

We analyzed five possible contextual and cultural moderating variables. Hypothesis 3 proposes that social needs attenuate the negative effect of crowding on satisfaction. Social needs predispose consumers to expect interaction with others and such needs are heightened in many contexts such as music festivals, sports events, and nightclubs. Congruent with $\mathrm{H} 3$, a high (vs. low) level of social needs attenuates the negative relationship between retail crowding and customer satisfaction $\left(r_{\text {high }}=.055 ; r_{\text {low }}=-.174 ; p<.05\right)$.

Hypothesis 4 postulates that in crowded retail environments, consumers with low (vs. high) perceived similarity will have higher levels of dissatisfaction. Crowd similarity reflects the focal consumer's identification with other consumers in the retail setting, thus reduced perceived similarity promotes dissatisfaction (Maner et al., 2007). Consistent $\mathrm{H} 4$ the findings indicate that high (vs. low) perceived similarity diminishes the negative effect of crowding on customer satisfaction $\left(r_{\text {high }}=-.106 ; r_{\text {low }}=-.410 ; p<.05\right)$.

Hypothesis 5 proposes a diminishing effect of crowd expectations on customer satisfaction. Crowd expectations are particularly salient in consumption situations or events linked to crowding, such as Black Friday and Christmas (Spangenberg et al., 2006). Our findings support that a diminishing effect of high (vs. low) crowd expectations on customer satisfaction $\left(r_{\text {high }}=-.017 ; r_{\text {low }}=-.308 ; p<.05\right)$. This finding supports H5.

Hypothesis 6 suggests that, in crowded retail environments, consumers with high time pressure will have higher levels of dissatisfaction than consumers with low time pressure. However, our findings fail to support $\mathrm{H6}\left(r_{h i g h}=-.485 ; r_{l o w}=-.109 ; p=n s\right)$, suggesting that time pressure is not a significant moderator of crowding effects on customer satisfaction. 
Regarding cultural moderators, hypothesis 7 proposes that, in crowded retail environments, consumers in cultures characterized by low (vs. high) uncertainty avoidance will have higher levels of dissatisfaction. Our results support such a prediction indicating that low uncertainty avoidance cultures yield a stronger effect $\left(r_{\text {High }}=-.128 ; r_{\text {Low }}=-.305 ; p<\right.$ .05). Alternative moderation analyses are presented in Appendix B1 and B2.

\section{Discussion and Implications}

During the past two decades academics have tried to understand retail crowding in several contexts. As a result, the crowding literature is quite fragmented (Mehta, 2013). To make sense of the mixed findings, we propose a robust theoretical model supported by an integrative analysis by Meta-Analytic Structural Equation Modeling (MASEM). Additionally, we investigate the mediating effect of negative emotions in the relationship between retail crowding and customer satisfaction. Our findings add new insight into the retail crowding literature (Mehta, 2013; Pons et al., 2016; Dahm et al., 2018; Park and Zhang, 2019; Blut and Iyer, 2020).

In this meta-analysis, crowding had a strong negative effect on customer satisfaction. Negative emotions associated with crowding explain this relationship. Perceived crowding easily induces negative mood, thus influencing consumers' shopping activities (e.g., Isen et al., 1978). Regarding the proposed moderators, uncertainty avoidance, crowding expectations, and perceived crowd similarity attenuate the negative relationship between crowding and satisfaction. Unexpectedly, time pressure did not influence the effect of retail crowding on customer satisfaction. A possible explanation is that only a handful of studies $(n=10)$ examined time pressure, thus limiting the power of our analyses. 


\subsection{Theoretical Implications}

Our integrative theoretical model of retail crowding contributes to the literature and retail practice in several ways. First, our findings confirm the mediating effect of negative emotions in the relationship between retail crowding and satisfaction. Eroglu et al. (2005) examined several discrete emotions (anger, disgust, and contempt) as mediators but their findings are not generalizable (Mehta, 2013). Consequently, our meta-analysis provides a more nuanced understanding of the role of negative emotions in crowded retail environments. Second, our moderation analyses provide a deeper understanding of when crowding influences consumer satisfaction (Pons et al., 2016). Although recent research has identified several important moderators (Blut and Iyer, 2020), their study focused on differences between human and spatial crowding. Our research thus advances prior literature by exploring new moderators.

In sum, we demonstrate the negative effect of retail crowding on consumer satisfaction, contributing to the retailing literature (Metha, 2013). Second, we show the mediating effect of negative emotions in explaining the impact of perceived crowding on satisfaction, thus enhancing prior retail crowding research (Machleit et al., 2000; Eroglu et al., 2005).

In terms of moderating effects, we found that cultures characterized by low (vs. high) uncertainty avoidance tend to exhibit higher levels of dissatisfaction in crowded stores. This finding contributes to previous studies on cultural effects (Metha, 2013; Das and Varshneya, 2014; Lucia-Palacios et al., 2018; Demoulin and Williams, 2019). We further demonstrate that two contextual factors, social interactions and identification with other consumers, attenuate the negative impact of crowding on satisfaction. Finally, time pressure did not failed to influence the effect of retail crowding on customer satisfaction. 


\subsection{Managerial Implications}

This paper also highlights some important managerial applications. For instance, managers could use effective strategies to attenuate the negative emotions caused by retail crowding, such as using social elements to diminish the consumers' negative emotions towards crowding situations (Demoulin and Willems, 2019).

First, the negative impact of crowding on satisfaction is likely to be magnified in shopping situations involving social interactions (e.g., restaurants). In such contexts, managers might want to focus on the spatial layout to reduce perceived crowding (Tombs and McColl-Kennedy, 2003). Another way to attenuate the negative effect of crowding is to use background music and other sensory cues in the retail environment (e.g., Herter et al., 2014).

Second, employing more salespeople could be effective in attenuating negative emotions caused by crowded stores. Previous research shows that social elements are one of the most important factors to diminish consumer irritation in crowded settings (Demoulin and Willems, 2019). Thus, retailers need to carefully manage the number of people in the store to reduce negative emotions and customer dissatisfaction.

Third, it is important to effectively manage the queuing process. For example, extending the opening hours might reduce long waits at the cash registrar. Or, retailers can install self-cash registers since this tool promoted better evaluation by consumers comparing to traditional queue systems (Dahm et al., 2018). Providing information about expected waiting times can also be helpful in managing queues.

Finally, retail managers could consider actions to evoke consumers identification or similarity perceptions (Boles et al., 2000). Merchandise such as t-shirts or baseball caps can 
be effective in increasing perceived similarity with other consumers, thus mitigating the negative effect of crowding on customer satisfaction.

\subsection{Limitations and Future Research}

Despite its contributions, this study has several limitations that can spur future research. For example, it would be useful to examine the effect of retail crowding on customer satisfaction with specific marketing stimuli such as background music, store design, salespeople persuasion, and self-checkout kiosks. It could also be interesting to examine potential differences in online and offline contexts. Future research is needed to better understand the relationship between retail crowding and firm outcomes (e.g., loyalty, wordof-mouth, financial performance).

Future research should also compare retail contexts (e.g., popular vs. sophisticated store/brand; promotion vs. non-promotion store/brand) to gain an in-depth understanding of retail crowding effects. Also, considering the shopping situation (time pressure; self-buying or

gift-buying) would be useful in advancing our understanding of positive or negative effects of retail crowding on customer satisfaction.

Finally, it would be useful to employ various research methodologies to enhance our understanding of the crowding effect. For instance, longitudinal studies would be helpful, especially given the current pandemic. Qualitative studies could also help explaining some conflicting findings that emerged in this research (e.g., time pressure).

\section{References}


Babić Rosario, A., Sotgiu, F., De Valck, K., and Bijmolt, T. H. (2016), “The effect of electronic word of mouth on sales: A meta-analytic review of platform, product, and metric factors”, Journal of Marketing Research, Vol. 53 No. 3, pp. 297-318.

Baker, J., and Wakefield, K. L. (2012), "How consumer shopping orientation influences perceived crowding, excitement, and stress at the mall ", Journal of the Academy of Marketing Science, Vol. 40 No. 6, pp. 791-806.

Blut, M., and Iyer, G. R. (2020), “Consequences of Perceived Crowding: A Meta-Analytical Perspective”, Journal of Retailing, in press.

Bressolles, G., Durrieu, F., and Giraud, M. (2007), “The impact of electronic service quality's dimensions on customer satisfaction and buying impulse”, Journal of Customer Behaviour, Vol. 6 No. 1, pp. 37-56.

Brocato, E. D., Voorhees, C. M., and Baker, J. (2012), "Understanding the influence of cues from other customers in the service experience: A scale development and validation”, Journal of Retailing, Vol. 88 No. 3, pp. 384-398.

Brocato, E., Voorhees, D.C.M., Baker, J., 2012. Understanding the influence of cues from other customers in the service experience: a scale development and validation ”, Journal of Retailing, Vol. 88, pp. 384-398.

Cavanaugh, L. A. (2014), “Because I (don't) deserve it: How relationship reminders and deservingness influence consumer indulgence”, Journal of Marketing Research, Vol. 51 No. 2, pp. 218-232.

Cheung, M. W. L. (2015), “metaSEM: An R package for meta-analysis using structural equation modeling", Frontiers in Psychology, Vol. 5, pp. 1521.

Dahm, M., Wentzel, D., Herzog, W., and Wiecek, A. (2018), “Breathing Down Your Neck!: The Impact of Queues on Customers Using a Retail Service”, Journal of Retailing, Vol. 94 No. 2, pp. 217-230. 
Das, G., and Varshneya, G. (2017), "Consumer emotions: Determinants and outcomes in a shopping mall”, Journal of Retailing and Consumer Services, Vol. 38, pp. 177-185.

Demoulin, N., and Willems, K. (2019), "Servicescape irritants and customer satisfaction: The moderating role of shopping motives and involvement", Journal of Business Research, Vol. 104, pp. 295-306.

Dion, D. (2004), "Personal control and coping with retail crowding", International Journal of Service Industry Management, Vol. 15 No. 3, pp. 250-263.

Eroglu, S. A., Machleit, K., and Barr, T. F. (2005), "Perceived retail crowding and shopping satisfaction: the role of shopping values”, Journal of business research, Vol. 58 No. 8, pp. 1146-1153.

Gelbrich, K., and Sattler, B. (2014), “Anxiety, crowding, and time pressure in public selfservice technology acceptance”, Journal of Services Marketing, Vol. 28 No. 1, pp. 8294.

Geyskens, I., Krishnan, R., Steenkamp, J. B. E., and Cunha, P. V. (2009), “A review and evaluation of meta-analysis practices in management research”, Journal of Management, Vol. 35 No. 2, pp. 393-419.

Harrell, G. D., Hutt, M. D., and Anderson, J. C. (1980), "Path analysis of buyer behavior under conditions of crowding”, Journal of Marketing Research, Vol. 17 No. 1, pp. 4551.

Hedges, L. V., and Olkin, I. (2014), "Statistical methods for meta-analysis”, Academic press.

Herter, M. M., dos Santos, C. P., and Pinto, D. C. (2014), ““"Man, I shop like a woman!” The effects of gender and emotions on consumer shopping behaviour outcomes”, International Journal of Retail and Distribution Management, Vol. 42 No. 9, pp. $780-804$. 
Hock, S ”, J., and Bagchi, R. (20187), “The impact of crowding on calorie consumption”, Journal of Consumer Research, Vol. 44 No. 5, pp. 1123-1140.

Hofstede, G. (2011), “Dimensionalizing cultures: the Hofstede model in context”, Online Readings in Psychology and Culture, Vol. 2, No. 1, pp. 8.

Hui, M. K., and Bateson, J. E. (1991), "Perceived control and the effects of crowding and consumer choice on the service experience”, Journal of Consumer Research, Vol. 18 No. 2, pp. 174-184.

Hunter, J. E., and Schmidt, F. L. (2004), “Methods of meta-analysis: Correcting error and bias in research findings", Sage.

Isen, A. M., and Simmonds, S. F. (1978), "The effect of feeling good on a helping task that is incompatible with good mood", Social Psychology, pp. 346-349.

Jak, S. (2015), “Meta-analytic structural equation modelling”, Dordrecht, Neth: Springer.

Kim, D. Y., Wen, L., and Doh, K. (2010), “Does cultural difference affect customer's response in a crowded restaurant environment? A comparison of American versus Chinese customers”, Journal of Hospitality and Tourism Research, Vol. 34 No. 1, pp. 103-123.

Knoeferle, K. M., Paus, V. C., and Vossen, A. (2017), “An upbeat crowd: fast in-store music alleviates negative effects of high social density on customers' spending”, Journal of Retailing, Vol. 93 No. 4, pp. 541-549.

Krohne, H. W., Hock, M., and Kohlmann, C. W. (1992), “Coping dispositions, uncertainty and emotional arousal (pp. 73-96), Johannes-Gutenberg-Univ., Abt. Persönlichkeitspsychologie.

Kuppens, P., Realo, A., and Diener, E. (2008), "The role of positive and negative emotions in life satisfaction judgment across nations”, Journal of personality and social psychology, Vol. 95 No. 1, pp. 66. 
Kwon, H., Ha, S., and Im, H. (2016), “The impact of perceived similarity to other customers on shopping mall satisfaction”, Journal of Retailing and Consumer Services, Vol. 28, pp. 304-309.

Lucia-Palacios, L., Pérez-López, R., and Polo-Redondo, Y. (2016), “Cognitive, affective and behavioural responses in mall experience: A qualitative approach”, International Journal of Retail and Distribution Management, Vol. 44 No. 1, pp. 4-21.

Lucia-Palacios, L., Pérez-López, R., and Polo-Redondo, Y. (2018), “Can social support alleviate stress while shopping in crowded retail environments? ", Journal of Business Research, Vol. 90, pp. 141-150.

Machleit, K. A., Eroglu, S. A., and Mantel, S. P. (2000), "Perceived retail crowding and shopping satisfaction: what modifies this relationship?", Journal of consumer psychology, Vol. 9 No. 1, pp. 29-42.

Mehta, R. (2013), “Understanding perceived retail crowding: A critical review and research agenda”, Journal of Retailing and Consumer Services, Vol. 20 No. 6, pp. 642-649.

Moher, D., Liberati, A., Tetzlaff, J., and Altman, D. G. (2009), "Preferred reporting items for systematic reviews and meta-analyses: the PRISMA statement", Annals of internal medicine, Vol. 151 No. 4, pp. 264-269.

Neale, M. C., Hunter, M. D., Pritikin, J. N., Zahery, M., Brick, T. R., Kirkpatrick, R. M., ... and Boker, S. M. (2016), “OpenMx 2.0: Extended structural equation and statistical modeling”, Psychometrika, Vol. 81 No. 2, pp. 535-549.

Nguyen, D. T., DeWitt, T., and Russell-Bennett, R. (2012), “Service convenience and social servicescape: retail vs hedonic setting”, Journal of Services Marketing, Vol. 26 No. 4, pp. $265-277$. 
Noone, B. M., and Mattila, A. S. (2009), "Consumer reaction to crowding for extended service encounters", Managing Service Quality: An International Journal. Vol. 19 No. 1, pp. 31-41.

Pan, Y., and Siemens, J. C. (2011), "The differential effects of retail density: An investigation of goods versus service settings", Journal of Business Research, Vol. 64 No. 2, pp. 105-112.

Pons, F., Giroux, M., Mourali, M., and Zins, M. (2016), “The relationship between density perceptions and satisfaction in the retail setting: Mediation and moderation effects”, Journal of Business Research, Vol. 69 No. 2, pp. 1000-1007.

Pons, F., Mourali, M., and Giroux, M. (2014), “The density-satisfaction relationship revisited: The role of scarcity and consumers affective reactions in a crowded retail situation”, Journal of Retailing and Consumer Services, Vol. 21 No. 1, pp. 54-60.

Qian, C., Chandrashekaran, M., and Yu, K. (2015), "Understanding the role of consumer heterogeneity in the formation of satisfaction uncertainty", Psychology and Marketing, Vol. 32 No. 1, pp. 78-93.

Sinha, S. P., and Nayyar, P. (2000), "Crowding effects of density and personal space requirements among older people: The impact of self-control and social support", The Journal of social psychology, Vol. 140 No. 6, pp. 721-728.

Spangenberg, E. R., Sprott, D. E., Grohmann, B., and Tracy, D. L. (2006), “Gender-congruent ambient scent influences on approach and avoidance behaviors in a retail store", Journal of Business Research, Vol. 59(12), pp. 1281-1287.

Thomas, V. L., and Saenger, C. (2019), "Feeling excluded? Join the crowd: How social exclusion affects approach behavior toward consumer-dense retail environments”, Journal of Business Research. 
Van Rompay, T”, J., Krooshoop, J., Verhoeven, J. W., and Pruyn, A. T. (2012), “With or without you: Interactive effects of retail density and need for affiliation on shopping pleasure and spending”, Journal of business research, Vol. 65 No. 8, pp. 1126-1131.

Viechtbauer, W. (2010), “Conducting meta-analyses in R with the metafor package”, Journal of statistical software, Vol. 36 No. 3, pp. 1-48.

Viswesvaran, C., \& Ones, D. S. (1995). Theory testing: Combining psychometric metaanalysis and structural equations modeling. Personnel psychology, 48(4), 865-885.

Wharton (2020), "How covid-19 will change the way we shop. Knowledge@ Wharton. Retrieved from https://knowledge.wharton.upenn.edu/article/covid-19-will-changeway-shop/

Wong, S., and Lau, E. (2001), "Understanding the behavior of Hong Kong Chinese tourists on group tour packages”, Journal of Travel Research, Vol. 40, pp. 57-67.

Wumei, L., Zengguang, M., and Xuhua, W. (2020), “A meta-analysis of the effect of crowding on consumers' emotional reactions and shopping-related behavioral reactions", Acta Psychologica Sinica, Vol. 2 No. 10, pp. 1237-1252. 